\title{
Warm gas accretion onto the Galaxy
}

\section{J. Bland-Hawthorn}

\author{
School of Physics, University of Sydney, Australia, NSW 2006
}

email: jbh@physics.usyd.edu.au

\begin{abstract}
We present evidence that the accretion of warm gas onto the Galaxy today is at least as important as cold gas accretion. For more than a decade, the source of the bright $\mathrm{H} \alpha$ emission (up to $750 \mathrm{mR} \dagger$ ) along the Magellanic Stream has remained a mystery. We present a hydrodynamical model that explains the known properties of the $\mathrm{H} \alpha$ emission and provides new insights on the lifetime of the Stream clouds. The upstream clouds are gradually disrupted due to their interaction with the hot halo gas. The clouds that follow plough into gas ablated from the upstream clouds, leading to shock ionisation at the leading edges of the downstream clouds. Since the following clouds also experience ablation, and weaker $\mathrm{H} \alpha(100-200 \mathrm{mR})$ is quite extensive, a disruptive cascade must be operating along much of the Stream. In order to light up much of the Stream as observed, it must have a small angle of attack $\left(\approx 20^{\circ}\right)$ to the halo, and this may already find support in new $\mathrm{HI}$ observations. Another prediction is that the Balmer ratio $(\mathrm{H} \alpha / \mathrm{H} \beta)$ will be substantially enhanced due to the slow shock; this will soon be tested by upcoming WHAM observations in Chile. We find that the clouds are evolving on timescales of 100-200 Myr, such that the Stream must be replenished by the Magellanic Clouds at a fairly constant rate $(\gtrsim 0.1$ $\left.\mathrm{M}_{\odot} \mathrm{yr}^{-1}\right)$. The ablated material falls onto the Galaxy as a warm drizzle; diffuse ionized gas at $10^{4} \mathrm{~K}$ is an important constituent of galactic accretion. The observed $\mathrm{H} \alpha$ emission provides a new constraint on the rate of disruption of the Stream and, consequently, the infall rate of metal-poor gas onto the Galaxy. We consider the stability of Hi clouds falling towards the Galactic disk and show that most of these must break down into smaller fragments that become partially ionized. The Galactic halo is expected to have huge numbers of smaller neutral and ionized fragments. When the ionized component of the infalling gas is accounted for, the rate of gas accretion is $\sim 0.4 \mathrm{M}_{\odot} \mathrm{yr}^{-1}$, roughly twice the rate deduced from $\mathrm{H}$ i observations alone.
\end{abstract}

Keywords. Galaxies: interaction, Magellanic Clouds - Galaxy: evolution - ISM: individual (Smith Cloud) - shock waves - instabilities - hydrodynamics

\section{Introduction}

It is now well established that the observed baryons over the electromagnetic spectrum account for only a fraction of the expected baryon content in Lambda Cold Dark Matter cosmology. This is true on scales of galaxies and, in particular, within the Galaxy where easily observable phases have been studied in great detail over many years. The expected baryon fraction $\left(\Omega_{B} / \Omega_{D M} \approx 0.17\right)$ of the dark halo mass $\left(1.4 \times 10^{12} \mathrm{M}_{\odot}\right.$; Smith et al. 2007$)$ leads to an expected baryon mass of $2.4 \times 10^{11} \mathrm{M}_{\odot}$ but a detailed inventory reveals only a quarter of this mass (Flynn et al. 2006 $\ddagger$ ). Moreover, the build-up of stars in the Galaxy requires an accretion rate of $1-3$ $\mathrm{M}_{\odot} \mathrm{yr}^{-1}$ (Williams \& McKee 1997; Binney et al. 2000), substantially larger than what can be accounted for from direct observation. We can extend the same argument to M31 where the total baryon mass is $\lesssim 10^{11} \mathrm{M}_{\odot}$ (Tamm et al. 2007). For the Galaxy, the predicted baryon mass may be a lower bound if the upward correction in the LMC-SMC orbit motion reflects a larger halo

$\dagger 1$ Rayleigh $(\mathrm{R})=10^{6} / 4 \pi$ photons $\mathrm{cm}^{-2} \mathrm{~s}^{-1} \mathrm{sr}^{-1}$, equivalent to $5.7 \times 10^{-18} \mathrm{erg} \mathrm{cm}^{-2} \mathrm{~s}^{-1}$ $\operatorname{arcsec}^{-2}$ at $\mathrm{H} \alpha$.

$\ddagger$ A decade ago, it was claimed that MACHOs may be important in the halo but these can only make up a negligible fraction by mass (Tisserand et al. 2007). 
mass (Kallivayalil et al. 2006; Piatek et al. 2008; cf. Wilkinson \& Evans 1999). Taken together, these statements suggest that most of the baryons on scales of galaxies have yet to be observed.

So how do galaxies accrete their gas? Is the infalling gas confined by dark matter? Does the gas arrive cold, warm or hot? Does the gas rain out of the halo onto the disk or is it forced out by the strong disk-halo interaction? These issues have never been resolved, either through observation or through numerical simulation. Hi observations of the nearby universe suggest that galaxy mergers and collisions are an important aspect of this process (Hibbard \& van Gorkom 1996), but tidal interactions do not guarantee that the gas settles to one or other galaxy. The most spectacular interaction phenomenon is the Magellanic Hi Stream that trails from the LMC-SMC system (10:1 mass ratio) in orbit about the Galaxy. Since its discovery in the 1970 s, there have been repeated attempts to explain the Stream in terms of tidal and/or viscous forces (q.v. Mastropietro et al. 2005; Connors et al. 2005). Indeed, the Stream has become a benchmark against which to judge the credibility of N-body+gas codes in explaining gas processes in galaxies. A fully consistent model of the Stream continues to elude even the most sophisticated codes.

Here, we demonstrate that $\mathrm{H} \alpha$ detections along the Stream (Fig. 1) are providing new insights on the present state and evolution of the Hi gas. At a distance of $D \approx 55 \mathrm{kpc}$, the expected $\mathrm{H} \alpha$ signal excited by the cosmic and Galactic UV backgrounds are about $3 \mathrm{mR}$ and $25 \mathrm{mR}$ respectively (Bland-Hawthorn \& Maloney 1999, 2002), significantly lower than the mean signal of 100-200 mR, and much lower than the few bright detections in the range $400-750 \mathrm{mR}$ (Weiner, Vogel \& Williams 2002). This signal cannot have a stellar origin since repeated attempts to detect stars along the Stream have failed.

Some of the Stream clouds exhibit compression fronts and head-tail morphologies (Brüns et al. 2005) and this is suggestive of confinement by a tenuous external medium. But the cloud:halo density ratio $\left(\eta=\rho_{c} / \rho_{h}\right)$ necessary for confinement can be orders of magnitude larger than that required to achieve shock-induced $\mathrm{H} \alpha$ emission (e.g. Quilis \& Moore 2001). Indeed, the best estimates of the halo density at the distance of the Stream $\left(\rho_{h} \sim 10^{-4} \mathrm{~cm}^{-3}\right.$; Bregman 2007) are far too tenuous to induce strong $\mathrm{H} \alpha$ emission at a cloud face. It is therefore surprising to discover that the brightest $\mathrm{H} \alpha$ detections lie at the leading edges of Hi clouds (Weiner et al. 2002) and thus appear to indicate that shock processes are somehow involved.

We summarize a model, first presented in Bland-Hawthorn et al. (2007), that goes a long way towards explaining the $\mathrm{H} \alpha$ mystery. The basic premise is that a tenuous external medium not only confines clouds, but also disrupts them with the passage of time. The growth time for Kelvin-Helmholtz $(\mathrm{KH})$ instabilities is given by $\tau_{\mathrm{KH}} \approx \lambda \eta^{0.5} / v_{h}$ where $\lambda$ is the wavelength of the growing mode, and $v_{h}$ is the apparent speed of the halo medium $\left(v_{h} \approx 350 \mathrm{~km} \mathrm{~s}^{-1}\right.$; see $\left.\S 2\right)$. At the distance of the Stream, the expected timescale for KH instabilities is less than for RayleighTaylor (RT) instabilities (see $\S 3$ ). For cloud sizes of order a few kiloparsecs and $\xi \approx 10^{4}$, the KH timescale can be much less than an orbital time $\left(\tau_{\mathrm{MS}} \approx 2 \pi D / v_{h} \approx 1 \mathrm{Gyr}\right)$. Once an upstream cloud becomes disrupted, the fragments are slowed with respect to the LMC-SMC orbital speed and are subsequently ploughed into by the following clouds.

In $\S 2$, the new hydrodynamical models are described and the results are presented; we discuss the implications of our model and suggest avenues for future research. In $\S 3$, we discuss the stability of $\mathrm{HI}$ clouds (high velocity clouds) moving through the corona toward the Galactic disk and briefly consider the Smith Cloud, arguably the HVC with the best observed kinematic and photometric parameters.

\section{A new hydrodynamical model}

There have been many attempts to understand how gas clouds interact with an ambient medium (Murray, White \& Blondin 1993; Klein, McKee \& Colella 1994). In order to capture the evolution of a system involving instabilities with large density gradients correctly, grid based methods (Liska \& Wendroff 1999; Agertz et al. 2007) are favoured over other schemes (e.g. Smooth Particle Hydrodynamics). We have therefore investigated the dynamics of the Magellanic Stream with two independent hydrodynamics codes, Fyris (Sutherland 2008) and 
Ramses (Teyssier 2002), that solve the equations of gas dynamics with adaptive mesh refinement. The results shown here are from the Fyris code because it includes non-equilibrium ionization, but we get comparable gas evolution from either code $\dagger$.

The brightest emission is found along the leading edges of clouds MS II, III and IV with values as high as $750 \mathrm{mR}$ for MS II. The $\mathrm{H} \alpha$ line emission is clearly resolved at $20-30 \mathrm{~km} \mathrm{~s}^{-1}$ FWHM, and shares the same radial velocity as the $\mathrm{HI}$ emission within the measurement errors (Weiner et al. 2002; Madsen et al. 2002). This provides an important constraint on the physical processes involved in exciting the Balmer emission.

In order to explain the $\mathrm{H} \alpha$ detections along the Stream, we concentrate our efforts on the disruption of the clouds labelled MS I-IV (Brüns et al. 2005). The Stream is trailing the LMCSMC system in a counter-clockwise, near-polar orbit as viewed from the Sun. The gas appears to extend from the LMC dislodged through tidal disruption although some contribution from drag must also be operating (Moore \& Davis 1994). Recently, the Hubble Space Telescope has determined an orbital velocity of $378 \pm 18 \mathrm{~km} \mathrm{~s}^{-1}$ for the LMC. While this is higher than earlier claims, the result has been confirmed by independent researchers (Piatek et al. 2008). Besla et al. (2007) conclude that the origin of the Stream may no longer be adequately explained with existing numerical models. The Stream velocity along its orbit must be comparable to the motion of the LMC; we adopt a value of $v_{\mathrm{MS}} \approx 350 \mathrm{~km} \mathrm{~s}^{-1}$.

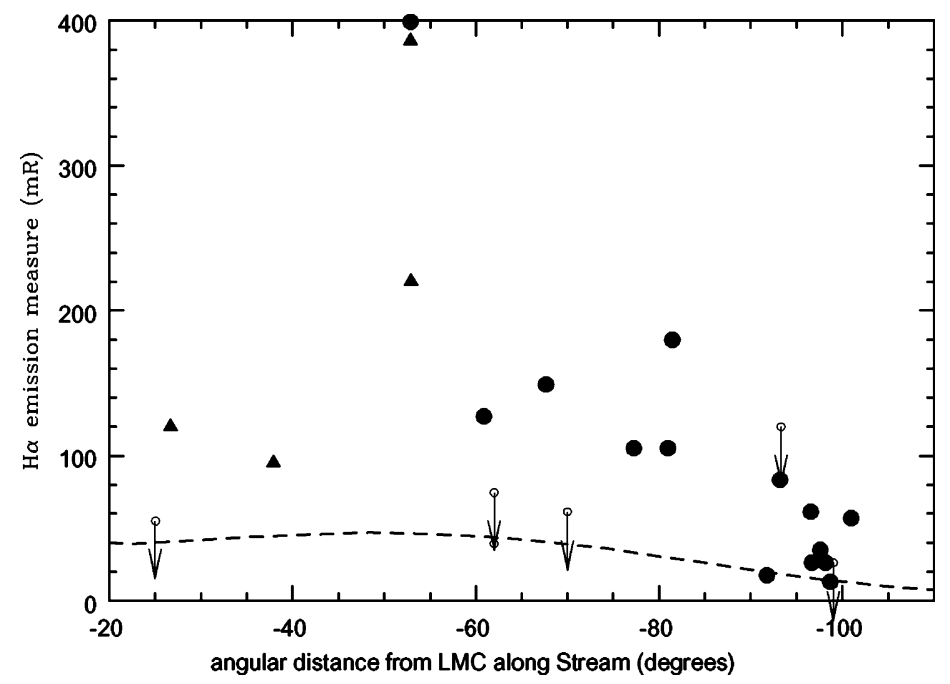

Figure 1. $\mathrm{H} \alpha$ measurements and upper limits along the Stream. The filled circles are from the WHAM survey by Madsen et al. (2002); the filled triangles are from the TAURUS survey by Putman et al. (2003). The dashed line model is the $\mathrm{H} \alpha$ emission measure induced by the ionizing intensity of the Galactic disk (Bland-Hawthorn \& Maloney 1999; 2002); this fails to match the Stream's $\mathrm{H} \alpha$ surface brightness by at least a factor of 3 .

\subsection{Model parameters}

Here we employ a 3D Cartesian grid with dimensions $18 \times 9 \times 9 \mathrm{kpc}[(x, y, z)=(432,216,216)$ cells] to model a section of the Stream where $x$ is directed along the Stream arc and the $z$ axis points towards the observer. The grid is initially filled with two gas components. The first is a hot thin medium representing the halo corona.

Embedded in the hot halo is (initially) cold Hi material with a total Hi mass of $3 \times 10^{7} \mathrm{M}_{\odot}$. The cold gas has a fractal distribution and is initially confined to a cylinder with a diameter of $4 \mathrm{kpc}$ and length $18 \mathrm{kpc}$ (Fig. 5); the mean volume and column densities are $0.02 \mathrm{~cm}^{-3}$

$\dagger$ Further details on the codes and comparative simulations are provided at http://www.aao.gov.au/astro/MS. 


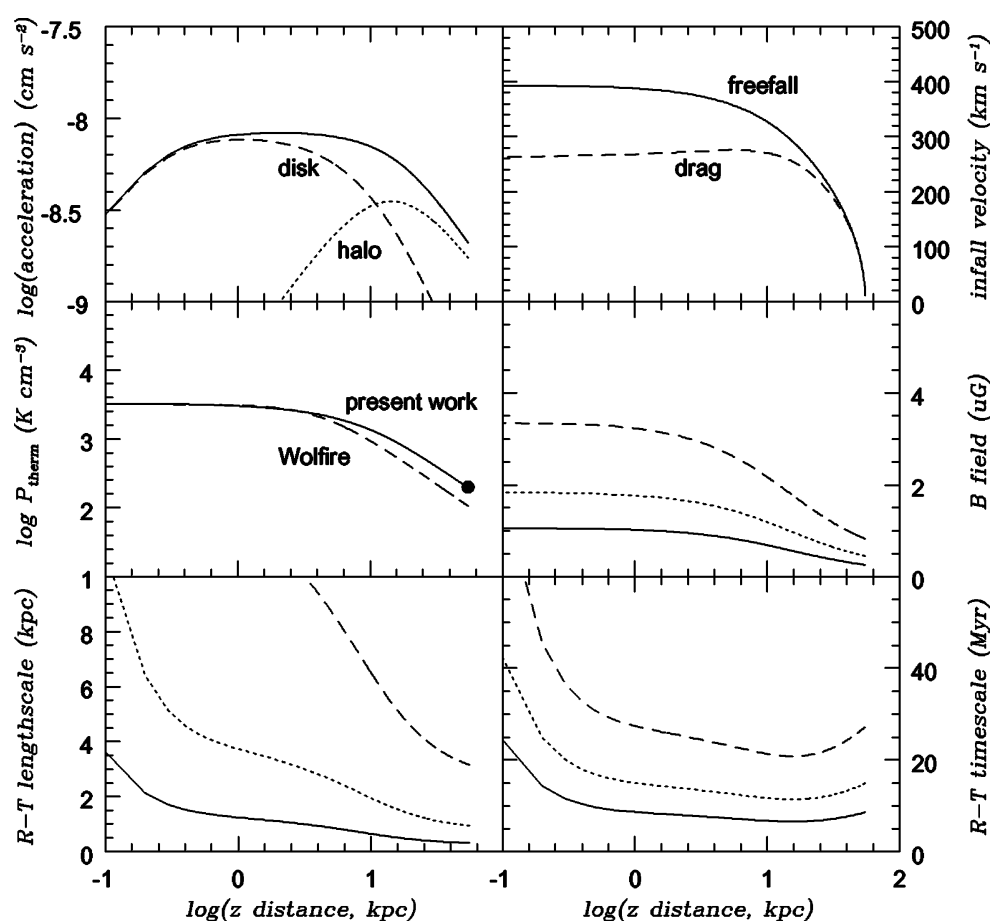

Figure 2. (a) gravitational acceleration due to the disk+halo - all plots are shown as a function of vertical height above the disk at the Solar Circle; (b) infall velocity for a point mass starting from the Magellanic Stream with halo drag $\left(C_{D}=1\right.$ discussed in $\S 3$; dashed curve) and without (solid); (c) coronal gas pressure for our model (solid line) compared to the Wolfire model (dashed) - the dot indicates the halo pressure used in our hydrodynamical model; (d) magnetic field strength for $\beta=1$ (dashed), $\beta=0.3$ (dotted), $\beta=0.1$ (solid); (e) minimum lengthscale for RT instability (discussed in $\S 3$ ); (e) timescale for RT instability (discussed in $\S 3$ ).

and $2 \times 10^{19} \mathrm{~cm}^{-2}$ respectively. The 3D spatial power spectrum $\left(P(k) \propto k^{-5 / 3}\right)$ describes a Kolmogorov turbulent medium with a minimum wavenumber $k$ corresponding to a spatial scale of $2.25 \mathrm{kpc}$, comparable to the size of observed clouds along the Stream.

We consider the hot corona to be an isothermal gas in hydrostatic equilibrium with the gravitational potential, $\phi(R, z)$, where $R$ is the Galactocentric radius and $z$ is the vertical scale height. We adopt a total potential of the form $\phi=\phi_{d}+\phi_{h}$ for the disk and halo respectively; for our calculations at the Solar Circle, we ignore the Galactic bulge. The galaxy potential is defined by

$$
\begin{aligned}
& \phi_{d}(R, z)=-c_{d} v_{\text {circ }}^{2} /\left(R^{2}+\left(a_{d}+\sqrt{z^{2}+b_{d}^{2}}\right)^{2}\right)^{0.5} \\
& \phi_{h}(R, z)=c_{h} v_{\text {circ }}^{2} \ln ((\psi-1) /(\psi+1))
\end{aligned}
$$

and $\psi=\left(1+\left(a_{h}^{2}+R^{2}+z^{2}\right) / r_{h}^{2}\right)^{0.5}$. The scaling constants are $\left(a_{d}, b_{d}, c_{d}\right)=(6.5,0.26,8.9) \mathrm{kpc}$ and $\left(a_{h}, r_{h}\right)=(12,210) \mathrm{kpc}$ with $c_{h}=0.33$ (e.g. Miyamoto \& Nagai 1975; Wolfire et al. 1995). The circular velocity $v_{\text {circ }} \approx 220 \mathrm{~km} \mathrm{~s}^{-1}$ is now well established through wide-field stellar surveys (Smith et al. 2007).

We determine the vertical acceleration at the Solar Circle using $g=-\partial \phi\left(R_{o}, z\right) / \partial z$ with $R_{o}=8 \mathrm{kpc}$. The hydrostatic halo pressure follows from

$$
\frac{\partial \phi}{\partial z}=-\frac{1}{\rho_{h}} \frac{\partial P}{\partial z}
$$


After Ferrara \& Field (1994), we adopt a solution of the form $P_{h}(z)=P_{o} \exp \left(\left(\phi\left(R_{o}, z\right)-\right.\right.$ $\left.\left.\phi\left(R_{o}, 0\right)\right) / \sigma_{h}^{2}\right)$ where $\sigma_{h}$ is the isothermal sound speed of the hot corona. To arrive at $P_{o}$, we adopt a coronal halo density of $n_{e, h}=10^{-4} \mathrm{~cm}^{-3}$ at the Stream distance $(55 \mathrm{kpc})$ in order to explain the Magellanic Stream $\mathrm{H} \alpha$ emission (Bland-Hawthorn et al. 2007), although this is uncertain to a factor of a few. We choose $T_{h}=2 \times 10^{6} \mathrm{~K}$ to ensure that OVI is not seen in the diffuse corona consistent with observation (Sembach et al. 2003); this is consistent with a rigorously isothermal halo for the Galaxy. Our solution to equation (2.3) is shown in Fig. 2(c) and it compares favorably with the pressure profile derive by others (e.g. Wolfire et al. 1995; Sternberg, McKee \& Wolfire 2002).

A key parameter of the models is the ratio of the cloud to halo pressure, $\xi=P_{c} / P_{h}$. If the cloud is to survive the impact of the hot halo, then $\xi \gtrsim 1$. A shocked cloud is destroyed in about the time it takes for the internal cloud shock to cross the cloud, during which time the cool material mixes and ablates into the gas streaming past. Only massive clouds with dense cores can survive the powerful shocks. An approximate lifetime $\dagger$ for a spherical cloud of diameter $d_{c}$ is

$$
\tau_{\mathrm{c}}=60\left(d_{c} / 2 \mathrm{kpc}\right)\left(v_{\mathrm{h}} / 350 \mathrm{kms}^{-1}\right)^{-1}(\eta / 100)^{0.5} \mathrm{Myr} .
$$

For $\eta$ in the range of $100-1000$, this corresponds to $60-180$ Myr for individual clouds. With a view to explaining the $\mathrm{H} \alpha$ observations, we focus our simulations on the lower end of this range.

For low $\eta$, the density of the hot medium is $n_{h}=2 \times 10^{-4} \mathrm{~cm}^{-3}$. The simulations are undertaken in the frame of the cold Hi clouds, so the halo gas is given an initial transverse velocity of $350 \mathrm{~km} \mathrm{~s}^{-1}$. The observations reveal that the mean $\mathrm{H} \alpha$ emission has a slow trend along the Stream which requires the Stream to move through the halo at a small angle of attack $\left(20^{\circ}\right)$ in the plane of the sky (see Fig. 5). Independent evidence for this appears to come from a wake of low column clouds along the Stream (Westmeier \& Koribalski 2008). Thus, the velocity of the hot gas as seen by the Stream is $\left(v_{x}, v_{y}\right)=(-330,-141) \mathrm{km} \mathrm{s}^{-1}$. The adiabatic sound speed of the halo gas is $200 \mathrm{~km} \mathrm{~s}^{-1}$, such that the drift velocity is mildly supersonic (transsonic), with a Mach number of 1.75 .

A unique feature of the Fyris simulations is that they include non-equilibrium cooling through time-dependent ionisation calculations (cf. Rosen \& Smith 2004). When shocks occur within the inviscid fluid, the jump shock conditions are solved across the discontinuity. This allows us to calculate the Balmer emission produced in shocks and additionally from turbulent mixing along the Stream (e.g. Slavin et al. 1993). We adopt a conservative value for the gas metallicity of $[\mathrm{Fe} / \mathrm{H}]=-1.0$ (cf. Gibson et al. 2000); a higher value accentuates the cooling and results in denser gas, and therefore stronger $\mathrm{H} \alpha$ emission along the Stream.

\subsection{Results}

The results of the simulations are shown in Figs. 3 - 5; we provide animations of the disrupting stream at http://www.aao.gov.au/astro/MS. In our model, the fractal Stream experiences a "hot wind" moving in the opposite direction. The sides of the Stream clouds are subject to gas ablation via KH instabilities due to the reduced pressure (Bernouilli's theorem). The ablated gas is slowed dramatically by the hot wind and is transported behind the cloud. As higher order modes grow, the fundamental mode associated with the cloud size will eventually fragment it. The ablated gas now plays the role of a "cool wind" that is swept up by the pursuing clouds leading to shock ionization and ablation of the downstream clouds. The newly ablated material continues the trend along the length of the Stream. The pursuing gas cloud transfers momentum to the ablated upstream gas and accelerates it; this results in Rayleigh-Taylor (RT) instabilities, especially at the stagnation point in the front of the cloud. We rapidly approach a nonlinear regime where the $\mathrm{KH}$ and $\mathrm{RT}$ instabilities become strongly entangled, and the internal motions become highly turbulent. The simulations track the progression of the shock fronts as they propagate into the cloudlets.

In Fig. 3, we show the predicted conversion of neutral to ionized hydrogen due largely to cascading shocks along the Stream. The drift of the peak to higher columns is due to the shocks

$\dagger$ Here we correct a typo in equation (1) of Bland-Hawthorn et al. (2007). 


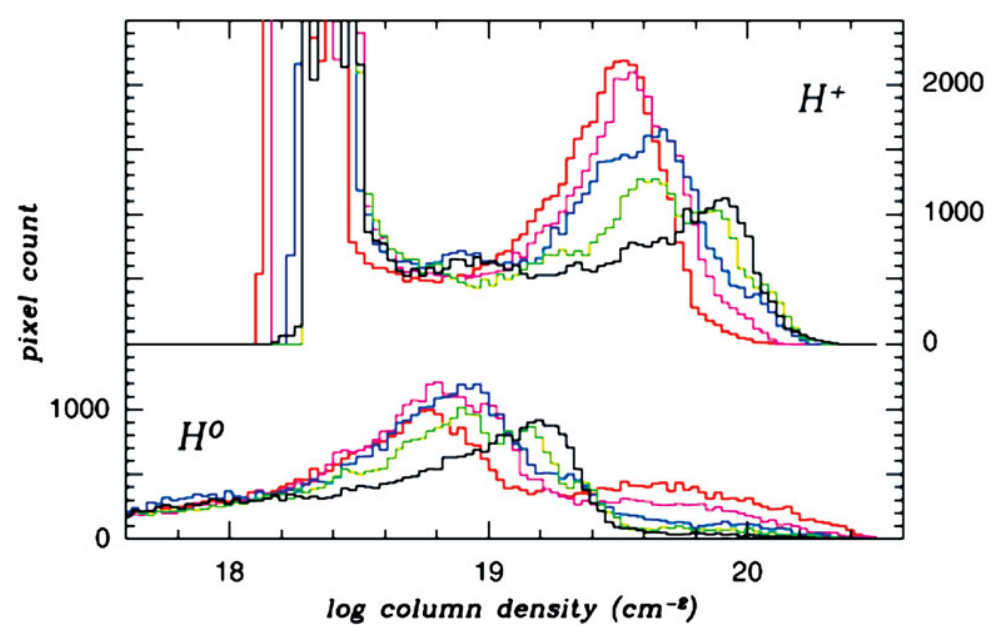

Figure 3. The dependence of the evolving fractions of $\mathrm{H}^{\circ}$ and $\mathrm{H}^{+}$on column density as the shock cascade progresses. The timesteps are 70 (red), 120 (magenta), 170 (blue), 220 (green) and $270 \mathrm{Myr}$ (black). The lowest column Hi becomes progressively more compressed with time but the highest column $\mathrm{HI}_{\mathrm{I}}$ is shredded in the cascade process; the fraction of ionized gas increases with time. The pile-up of electrons at low column densities arises from the x-ray halo.

eroding away the outer layers, thereby progressing into increasingly dense cloud cores. The ablated gas drives a shock into the Hi material with a shock speed of $v_{s}$ measured in the cloud frame. At the shock interface, once ram-pressure equilibrium is reached, we find $v_{s} \approx v_{\mathrm{h}} \eta^{-0.5}$. In order to produce significant $\mathrm{H} \alpha$ emission, $v_{s} \gtrsim 35 \mathrm{~km} \mathrm{~s}^{-1}$ such that $\eta \lesssim 100$. In Fig. 4 , we see the steady rise in $\mathrm{H} \alpha$ emission along the Stream, reaching $100-200 \mathrm{mR}$ after $120 \mathrm{Myr}$, and the most extreme observed values after $170 \mathrm{Myr}$. The power-law decline to bright emission measures is a direct consequence of the shock cascade. The shock-induced ionization rate is $1.5 \times 10^{47}$ phot $\mathrm{s}^{-1} \mathrm{kpc}^{-1}$. The predicted luminosity-weighted line widths of $20 \mathrm{~km} \mathrm{~s}^{-1}$ FWHM (Fig. 4, inset) are consistent with the $\mathrm{H} \alpha$ kinematics. In Fig. 5, the $\mathrm{H} \alpha$ emission is superimposed onto the projected Hi emission: much of it lies at the leading edges of clouds, although there are occasional cloudlets where ionized gas dominates over the neutral column. Some of the brightest emission peaks appear to be due to limb brightening, while others arise from chance alignments.

The simulations track the degree of turbulent mixing between the hot and cool media brought on by KH instabilities (e.g. Kahn 1980). The turbulent layer grows as the flow develops, mixing up hot and cool gas at a characteristic temperature of about $10^{4} \mathrm{~K}$. In certain situations, a sizeable H $\alpha$ luminosity can be generated (e.g. Canto \& Raga 1991) and the expected line widths are comparable to those observed in the Stream. Indeed, the simulations reveal that the fractal clouds develop a warm ionized skin along the entire length of the Stream. But the characteristic $\mathrm{H} \alpha$ emission (denoted by the shifting peak in Fig. 4) is comparable to the fluorescence excited by the Galactic UV field (Bland-Hawthorn \& Maloney 2002). We note with interest that narrow Balmer lines can arise from pre-cursor shocks (e.g. Heng \& McCray 2007), but these require conditions that are unlikely to be operating along the Stream.

\subsection{Discussion}

We have seen that the brightest $\mathrm{H} \alpha$ emission along the Stream can be understood in terms of shock ionization and heating in a transsonic (low Mach number) flow. For the first time, the Balmer emission (and associated emission lines) provides diagnostic information at any position along the Stream that is independent of the Hi observations. Slow Balmer-dominated shocks of this kind (e.g. Chevalier \& Raymond 1978) produce partially ionized media where a significant fraction of the $\mathrm{H} \alpha$ emission is due to collisional excitation. This can lead to Balmer decrements $(\mathrm{H} \alpha / \mathrm{H} \beta$ ratio $)$ in excess of 4 , i.e. significantly enhanced over the pure recombination ratio of about 3 , that will be fairly straightforward to verify in the brightest regions of the Stream. 


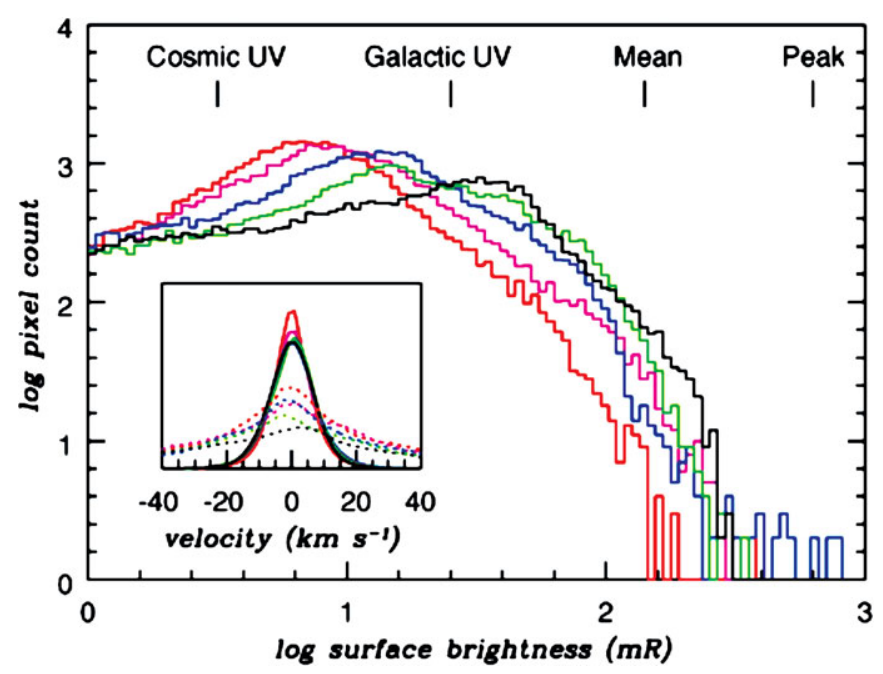

Figure 4. The evolving distribution of projected $\mathrm{H} \alpha$ emission as the shock cascade progresses. The timesteps are explained in Fig. 3. The extreme emission measures increase with time and reach the observed mean values after $120 \mathrm{Myr}$; this trend in brightness arises because denser material is ablated as the cascade evolves. The mean and peak emission measures along the Stream are indicated, along with the approximate contributions from the cosmic and Galactic UV backgrounds. Inset: The evolving $\mathrm{H} \alpha$ line width as the shock cascade progresses; the velocity scale is with respect to the reference frame of the initial Hi gas. The solid lines are flux-weighted line profiles; the dashed lines are volume-weighted profiles that reveal more extreme kinematics at the lowest densities.

The shock models predict a range of low-ionization emission lines (e.g. OI, SII), some of which will be detectable even though suppressed by the low gas-phase metallicity. There are likely to be EUV absorption-line diagnostics through the shock interfaces revealing more extreme kinematics (Fig. 4, inset), but these detections (e.g. OVI) are only possible towards fortuitous background sources (Sembach et al. 2001; Bregman 2007). The predicted EUV/x-ray emissivity from the post-shock regions is much too low to be detected in emission.

The characteristic timescale for large changes is roughly 100-200 Myr, and so the Stream needs to be replenished by the outer disk of the LMC at a fairly constant rate (e.g. Mastropietro et al. 2005). The timescale can be extended with larger $\eta$ values (equation (2.4)), but at the expense of substantially diminished $\mathrm{H} \alpha$ surface brightness. In this respect, we consider $\eta$ to be fairly well bounded by observation and theory.

What happens to the gas shedded from the dense clouds? Much of the diffuse gas will become mixed with the hot halo gas suggesting a warm accretion towards the inner Galactic halo. If most of the Stream gas enters the Galaxy via this process, the derived gas accretion rate is $\sim 0.4 \mathrm{M}_{\odot} \mathrm{yr}^{-1}$. The higher value compared to $\mathrm{H}_{\mathrm{I}}$ (e.g. Peek et al. 2008) is due to the gas already shredded, not seen by radio telescopes now. In our model, the HVCs observed today are unlikely to have been dislodged from the Stream by the process described here. These may have come from an earlier stage of the LMC-SMC interaction with the outer disk of the Galaxy.

The "shock cascade" interpretation for the Stream clears up a nagging uncertainty about the $\mathrm{H} \alpha$ distance scale for high-velocity clouds. Bland-Hawthorn et al. (1998) first showed that distance limits to HVCs can be determined from their observed $\mathrm{H} \alpha$ strength due to ionization by the Galactic radiation field, now confirmed by clouds with reliable distance brackets from the stellar absorption line technique (Putman et al. 2003; Lockman et al. 2008; Wakker et al. 2007). HVCs have smaller kinetic energies compared to the Stream clouds, and their interactions with the halo gas are not expected to produce significant shock-induced or mixing layer $\mathrm{H} \alpha$ emission, thereby supporting the use of $\mathrm{H} \alpha$ as a crude distance indicator. 

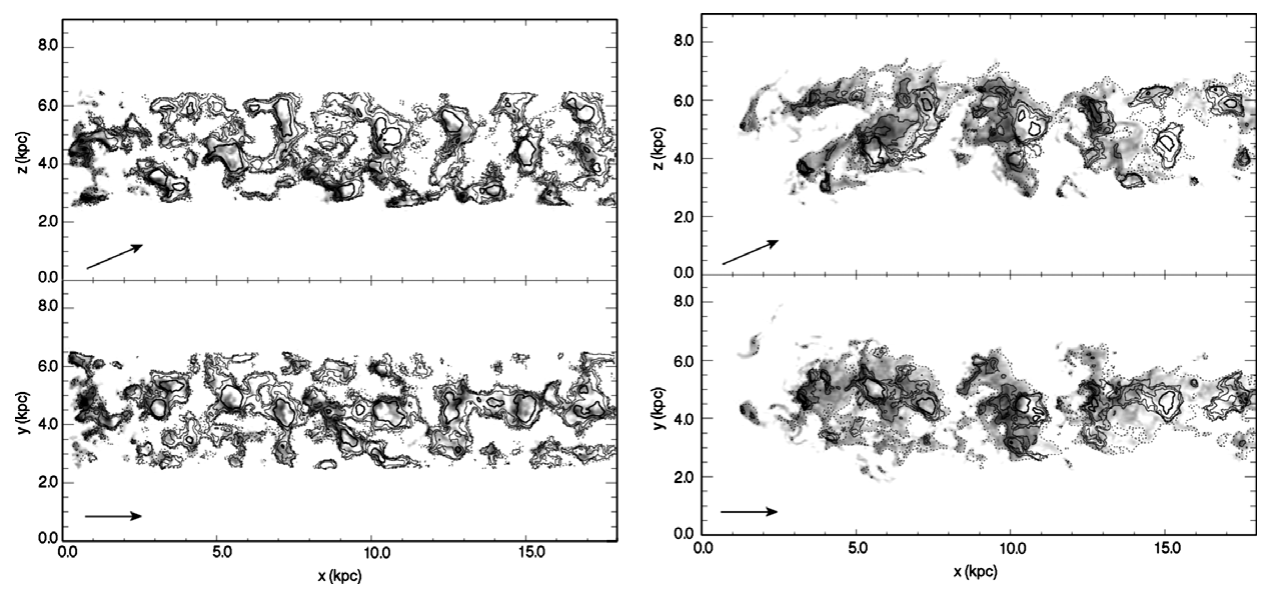

Figure 5. The initial fractal distribution of $\mathrm{HI}$ at $20 \mathrm{Myr}$, shown in contours, before the wind action has taken hold. The upper figure is in the $x-z$ frame as seen from above; the lower figure is the projected distribution on the plane of the sky $(x-y$ plane). Both distributions are integrated along the third axis. The logged Hi contours correspond to 18.5 (dotted), 19.0, 19.5, 20.0 , and 20.5 (heavy) $\mathrm{cm}^{-2}$. The greyscale shows weak levels of $\mathrm{H} \alpha$ along the Stream where black corresponds to $300 \mathrm{mR}$. The predicted $\mathrm{H}_{\mathrm{I}}$ (contours) and $\mathrm{H} \alpha$ (greyscale) distributions after 120 Myr. The angle of attack in the $[\mathrm{x}, \mathrm{y}, \mathrm{z}]$ coordinate frame is indicated. The $\mathrm{H} \alpha$ emission is largely, but not exclusively, associated with dense Hi gas.

Here, we have not attempted to reproduce the Hi observations of the Stream in detail. This is left to a subsequent paper where we explore a larger parameter space and include a more detailed comparison with the $\mathrm{H}_{\mathrm{I}}$ and $\mathrm{H} \alpha$ power spectrum, inter alia. We introduce additional physics, in particular, the rotation of the hot halo, a range of Stream orbits through the halo gas, and so on.

If we are to arrive at a satisfactory understanding of the Stream interaction with the halo, future deep $\mathrm{H} \alpha$ surveys will be essential. It is plausible that current $\mathrm{H} \alpha$ observations are still missing a substantial amount of gas, in contrast to the deepest Hi observations. We can compare the particle column density inferred from $\mathrm{HI}$ and $\mathrm{H} \alpha$ imaging surveys. The limiting $\mathrm{H}$ c column density is about $N_{H} \approx\left\langle n_{H}\right\rangle L \approx 10^{18} \mathrm{~cm}^{-2}$ where $\left\langle n_{H}\right\rangle$ is the mean atomic hydrogen density, and $L$ is the depth through the slab. By comparison, the $\mathrm{H} \alpha$ surface brightness can be expressed as an equivalent emission measure, $E_{m} \approx\left\langle n_{e}^{2}\right\rangle L \approx\left\langle n_{e}\right\rangle N_{e}$. Here $n_{e}$ and $N_{e}$ are the local and column electron density. The limiting value of $E_{m}$ in $\mathrm{H} \alpha$ imaging is about $100 \mathrm{mR}$, and therefore $N_{e} \approx 10^{18} /\left\langle n_{e}\right\rangle \mathrm{cm}^{-2}$. Whether the ionized and neutral gas are mixed or distinct, we can hide a lot more ionized gas below the imaging threshold for a fixed $L$, particularly if the gas is at low density $\left(\left\langle n_{e}\right\rangle \ll 0.1 \mathrm{~cm}^{-3}\right)$. A small or variable volume filling factor can complicate this picture but, in general, the ionized gas still wins out because of ionization of low density Hi by the cosmic UV background (Maloney 1993). In summary, even within the constraints of the cosmic microwave background (see Maloney \& Bland-Hawthorn 1999), a substantial fraction of the gas can be missed if it occupies a large volume in the form of a low density plasma (e.g. Rasmussen et al. 2003).

\section{Direct infall of $\mathrm{HI}$ onto the disk}

The conditions operating along the Magellanic Stream are unlikely to be representative of all Hi clouds that move through the Galactic corona. A related process is the infall of individual HI clouds towards the Galactic disk. The Galactic halo is home to many HVCs of unknown origin (Wakker 2001; Lockman et al. 2008). The survival and stability of these clouds is a problem that has long been recognized (e.g. Benjamin \& Danly 1997) which we now discuss.

It is likely that many or all halo clouds have experienced some deceleration during their transit through the lower halo. Using equation (2.3), we determine a freefall velocity for a cloud 
starting at the distance of the Stream (Fig. 1(b)) that is more than twice what is inferred for clouds at the Solar Circle (Wakker 2001), although some HVCs clearly have high space motions (e.g. Lockman et al. 2008). To explain this observation, Benjamin \& Danly (1997) investigated a drag equation for a cloud moving through a stationary medium,

$$
\mu_{c} \dot{v}_{c}=\frac{1}{2} C_{D} \rho_{h}(z) v_{c}^{2}-\mu_{c} g(z)
$$

where $\mu_{c}$ is the surface density of the cloud. Equation (3.1) only holds as long as the cloud stays together. The drag coefficient $C_{D}$ is a measure of the efficiency of momentum transfer to the cloud. For the high Reynolds numbers typical of astrophysical media, incompressible objects have $C_{D} \approx 0.4$ (e.g. a rough sphere) which indicates that the turbulent wake behind the plunging object efficiently transfers momentum to the braking medium. The leading face of a compressible cloud may become flattened, such that the approaching medium is brought to rest in the reference frame of the cloud; in this instance, $C_{D} \gtrsim 1$ may be more appropriate (we adopt $C_{D}=1$ here).

A solution specific to our model is shown in Fig. 2(b) where the freefall velocity is now slowed by about $35 \%$. In practice, the cloud's projected motion can be considerably less than its 3D space velocity (e.g. Lockman et al. 2008). In all likelihood, infalling HVCs have experienced significant deceleration through ram pressure exerted by the corona. But even before the cloud reaches terminal velocity, the cloud is expected to break up (Murray \& Lin 2004).

So how do clouds resist the destructive forces of RT and shock instabilities? In $\S 3.1$, we investigate the stabilizing influence of magnetic fields when a cloud passes through a magnetized medium. The halo magnetic field is poorly constrained at the present time (e.g. Sun et al. 2008). We describe the uniform magnetic field in terms of the pressure of the halo medium, or

$$
\frac{B^{2}}{8 \pi}=\beta P_{h}
$$

such that $B \approx 1 \mu \mathrm{G}$ at the distance of the Stream ( $55 \mathrm{kpc}$ ) if the field is in full equipartition with the corona (see Fig. 1(d)). But there is evidence that the field is weaker than implied here $(\beta \approx 0.3$; Sun et al. 2008), at least within $5 \mathrm{kpc}$ of the Galactic plane For the warm, denser lowlatitude gas (Reynolds layer), we adopt the new parameter fits of Gaensler et al. (2008) from a re-analysis of pulsar data. The lower $\beta$ value finds support from recent magnetohydrodynamic simulations of the Reynolds layer (Hill et al. 2008).

\subsection{Stability limits and growth timescales}

We consider the surface of a high velocity cloud as a boundary between two fluids. In practice, the Galactic ionizing radiation field imparts a multiphase structure to the cloud. At all galactic latitudes within the Stream distance, HVCs with column densities of order $10^{20} \mathrm{~cm}^{-2}$ or higher have partially ionized skins to a column depth of roughly $10^{19} \mathrm{~cm}^{-2}$ for sub-solar gas due to the Galactic ionizing field (see Bland-Hawthorn \& Maloney 1999; Wolfire et al. 2003). Between the warm ionized skin and the cool inner regions is a warm neutral medium of twice the skin thickness; both outer layers have a mean particle temperature of $\lesssim 10^{4} \mathrm{~K}$.

The cloud is denser than the halo gas. Because of the gravitational field, RT instabilities can grow on the boundary. Furthermore, KH instabilities may also develop due to the relative motion of the cloud with respect to an external medium. Recent work has shown that buoyant bubbles in galaxy clusters are stabilized against RT and KH instabilities by viscosity and surface tension due to magnetic fields in the boundary (De Young 2003; Kaiser et al. 2005; Jones \& De Young 2005). Here we examine whether HVC boundaries are similarly stabilized against disruption in the Galactic halo.

When there is no surface tension, no viscosity and no relative motion between the two media, the growth rate of the RT instability for a perturbation with wavenumber $k$ is $\omega=\sqrt{g k}$, where $g$ is the gravitational acceleration at the fluid boundary. The wavenumber is related to a perturbation length scale, $\ell=2 \pi / k$. The instability requires a few e-folding timescales to fully 
develop; the timescale is given by

$$
t_{\text {grow }}=\omega^{-1}=\sqrt{\frac{\ell}{2 \pi g}} .
$$

In the presence of a magnetic field, the transverse component $\left(B_{t r}\right)$ provides some surface tension which can help to suppress RT instabilities below a lengthscale of

$$
\ell_{\text {min }}=\frac{B_{t r}^{2}}{2 \rho_{c} g}
$$

(Chandrasekhar 1961). Here $\rho_{c}$ is the mass density of the denser medium, i.e. the cloud, and $B_{t r}$ is the average value of the transverse magnetic field at the boundary.

In order to illustrate when RT instabilities become important, we assume a flat rotation curve for the Galaxy (e.g. Binney \& Dehnen 1997)

$$
g \approx \frac{v_{\text {circ }}^{2}}{R}=1.6 \times 10^{-8}\left(\frac{v_{\text {circ }}}{220 \mathrm{~km} \mathrm{~s}^{-1}}\right)^{2}\left(\frac{R}{R_{o}}\right)^{-1} \mathrm{~cm} \mathrm{~s}^{-2}
$$

This is only a rough approximation to the form expected from equations (2.1) and (2.2). We stress that the actual behaviour discussed below, and shown in Figs. 2(e) and (f), solves for the gravitational potential correctly.

Shortly after the discovery of HVCs, it was thought that they may be self-gravitating. But this would place them at much greater distances than the Magellanic Stream (e.g. Oort 1966) which is now known not to be the case (e.g. Putman et al. 2003). Instead, we consider two cases: (i) HVCs in pressure equilibrium with the coronal gas; (ii) HVCs with parameters fixed by direct observation. In (i), because the temperature is not strongly dependent on radius, but the number density decreases rapidly with increasing radius, we expect the increased pressure to compress the clouds at lower latitudes.

We estimate the impact of RT instabilities using equations (2.3) and (3.3): for a cloud temperature of $T_{c}=10^{4} \mathrm{~K}$ (see $\S 2$ ) in pressure equilibrium with the hot halo, the electron density is given by

$$
\begin{aligned}
n_{e, c} & \approx n_{e, h} \frac{T_{h}}{T_{c}} \\
& =0.02\left(\frac{R}{55 \mathrm{kpc}}\right)^{-2}\left(\frac{T_{h}}{2 \times 10^{6} \mathrm{~K}}\right)\left(\frac{T_{c}}{10^{4} \mathrm{~K}}\right)^{-1} \mathrm{~cm}^{-3} .
\end{aligned}
$$

We use equations 3.4, 3.6 and 3.2 to estimate $\ell_{m i n}$ as a function of Galactocentric radius. The minimum length scale for instability is

$$
\begin{aligned}
\frac{\ell_{\min }}{R} & \sim \frac{8 \pi \beta k_{B} T_{c}}{m_{p} v_{\text {circ }}^{2}} \\
& =0.004\left(\frac{T_{c}}{10^{4} \mathrm{~K}}\right)\left(\frac{\beta}{0.1}\right)\left(\frac{v_{\text {circ }}}{220 \mathrm{~km} \mathrm{~s}^{-1}}\right)^{-2}
\end{aligned}
$$

and its associated growth timescale using equation (3.3)

$$
\begin{aligned}
t_{\text {grow }} & \sim \sqrt{\frac{4 \beta k_{B} T_{c}}{m_{p} v_{\text {circ }}^{2}}} \Omega^{-1} \\
& =1.1 \mathrm{Myr}\left(\frac{T_{c}}{10^{4} \mathrm{~K}}\right)^{\frac{1}{2}}\left(\frac{\beta}{0.1}\right)^{\frac{1}{2}}\left(\frac{v_{\text {circ }}}{220 \mathrm{~km} \mathrm{~s}^{-1}}\right)^{-2}\left(\frac{R}{R_{o}}\right)
\end{aligned}
$$

where the angular rotation rate is given by $\Omega=v_{\text {circ }} / R$.

Because we have assumed that $B^{2} \propto n_{e, h}$ (equipartition) and $n_{e, c} \propto n_{e, h}$ (pressure equilibrium), neither the minimum scale length or its growth timescale depend on the halo density or temperature. They do depend on the temperature of the clouds and the ionization state. If the clouds are hotter than $10^{4} \mathrm{~K}$, then $n_{e, c}$ is overestimated under the assumption of pressure 
equilibrium. This would lead to larger minimum instability lengthscales and growth timescales. If the Galactic rotation curve drops faster than the flat profile implied by equation (2.3), we would have underestimated both the minimum instability scale length and its associated growth timescale at large radii.

Under the assumption of pressure equilibrium, the falling clouds become more compressed as they approach the disk which can hasten cooling. This effect may help to stabilize against break up, particularly if a cool shell develops (cf. Sternberg \& Soker 2008).

In the absence of gravitational instability, the flow is stable against the KH instability if (Chandrasekhar 1961)

$$
U^{2}<\frac{B_{t r}^{2}\left(\rho_{c}+\rho_{h}\right)}{2 \pi \rho_{c} \rho_{h}}
$$

where $U$ is the relative velocity between the two fluids. When $\rho_{c}>\rho_{h}$, this requirement becomes

$$
\begin{aligned}
U & <\sqrt{\frac{8 \beta k_{B} T_{h}}{m_{p}}} \\
& =115\left(\frac{\beta}{0.1}\right)^{\frac{1}{2}}\left(\frac{T_{h}}{2 \times 10^{6} \mathrm{~K}}\right)^{\frac{1}{2}} \mathrm{~km} \mathrm{~s}^{-1}
\end{aligned}
$$

and we have described the magnetic field in terms of the halo pressure using equation 3.2. This requirement is also independent of the halo density as we have related the magnetic field to the halo pressure, although it is dependent on the halo temperature. This requirement is nearly satisfied for HVCs if the magnetic field is near equipartition.

\subsection{The Smith Cloud}

Arguably, the high-latitude $\mathrm{H}_{\mathrm{r}}$ cloud that we know most about is the Smith Cloud. Lockman et al. (2008) have recently published spectacular $\mathrm{HI}$ data for this $\mathrm{HVC}$ and deduce a remarkable amount about its past and future properties. The HVC has an estimated distance of $12.4 \pm 1.3$ kpc, a Galactocentric radius of $R \approx 8 \mathrm{kpc}$, a vertical height below the plane of $-2.9 \mathrm{kpc}$, a mass of at least $10^{6} M_{\odot}$ in a volume of order $3 \mathrm{kpc}^{3}$ corresponding to $n_{c} \approx 0.014 \mathrm{~cm}^{-3}$. The cloud has a prograde orbit that is inclined $30^{\circ}$ to the plane and appears to have come through the disk $70 \mathrm{Myr}$ ago at $R \approx 13 \mathrm{kpc}$ moving from above to below the plane.

In order to have punched through the disk, the shock crossing time for the cloud must be longer than for the disk. It can be shown that

$$
\frac{d_{c}}{z_{d}}>\sqrt{\frac{n_{d}}{n_{c}}}
$$

where $z_{d}$ is the vertical thickness and $n_{d}$ is the mean density of the Hi at the crossing point. This is essentially a statement that the surface density of the cloud must be higher than the disk. If we assume the cloud punched through the Galactic hydrogen density profile determined by Kalberla \& Dedes (2008), equation 3.11 indicates that the cloud was substantially thicker than the disk when it came through and somewhat more massive than what is observed now. Consistent with this picture, the observed wake may result from ablation processes induced by the impact. For cloudlets smaller than $100 \mathrm{pc}$, thermal conduction due to the halo corona (McKee \& Cowie 1977) and the Galactic radiation field convert the ablated gas to a clumpy plasma.

The kinetic energy of the Smith Cloud observed today is $\sim 10^{54}$ erg - this is enough to punch through the disk if sufficiently concentrated. Impulsive shock signatures at UV to x-ray wavelengths will have largely faded away, and the $\mathrm{Hr}$ "hole" at the crossing point will have been substantially stretched by differential shear $\dagger$.

$\dagger$ It is sometimes claimed, this meeting notwithstanding, that outer disk $\mathrm{HI}_{\mathrm{I}}$ "holes" are evidence of dark matter minihalos passing through the disk, but it can be shown that the gravitational impulse has negligible impact on the gaseous disk. 
Figs. 2(e) and (f) show that a cloud of several kpc can survive RT instabilities at these latitudes, but it is difficult to see how the Smith Cloud, like several other large HVCs, could have come in from, say, the distance of the Magellanic Stream. Lockman et al. (2008) use essentially the same Galactic potential as described here to determine the cloud's orbit parameters. We conjecture that either the cloud has been dislodged from the outer disk by a passing dwarf, or the cloud has been brought in by a confining dwarf potential. A cloud metallicity of $[\mathrm{Fe} / \mathrm{H}] \approx-1$ is appropriate in either scenario. Interestingly, the impulse from the Galactic disk can cause the gas to become dislodged from the confining dark halo or to oscillate within it. The interloper must be on a prograde orbit which rules out some infalling dwarfs (e.g. $\omega$ Cen; Bekki \& Freeman 2007), but conceivably implicates disrupting dwarfs like Canis Major or Sagittarius, assuming these were still losing gas in the recent past.

\subsection{Discussion}

In $\S 2$, we presented evidence for a shock cascade along the Magellanic Stream arising from the disruption of upstream clouds due to their interaction with the Galactic halo. Bland-Hawthorn et al. (2007) make firm predictions that can be tested in future observations. A possible improvement is to consider the entire Magellanic System, i.e. the influence of the LMC-SMC system that lies further upstream. Mastropietro et al. (2008) present evidence for a strong interaction along the leading edge of the LMC; for their quoted model parameters, it seems plausible that this results in a stand-off bowshock ahead of the galaxy. The cross wind over the face of the LMC could be confused for a starburst-driven wind from the LMC (cf. Lehner \& Howk 2007). In all likelihood, the LMC-SMC system creates a turbulent wake behind it which may impact the development of instabilities in the trailing stream.

The issue of cloud survival is highly complex. In $\S 3$, we did not consider the role of viscosity in quenching RT or KH instabilities. Simulations have shown that viscosity does lead to stabilization (Pavlovski et al. 2008) but we have not been able to estimate a lengthscale or a growth timescale appropriate for our setting. Kaiser et al. (2005) show that when the density ratio between the two media is large, $\mathrm{KH}$ instabilities fail to grow and the growth rate of RT instabilities depends only on the properties of higher density medium, in our case the cloud medium. However their result, taken in the limit of one density much larger than the other, $\rho_{2} \gg \rho_{1}$, will not apply if $\nu_{1} \rho_{1} \gg \nu_{2} \rho_{2}$. Here the subscripts refer to the fluids on either side of the boundary and $\nu$ is the kinematic viscosity. Because diffusivity coefficients are sensitive functions of temperature $\left(\propto T^{-2.5}\right)$, they could dampen fluid instabilities. Unfortunately the expected differences in temperatures between HVCs and the halo gas (corona) suggest that $\nu_{1} \rho_{1} \gg \nu_{2} \rho_{2}$ and thus we cannot apply the limit used by Kaiser et al. (2005). A proper treatment is required to cover the Galactic halo setting.

Other studies have argued that the KH instability leads to a turbulent mixing layer on the surface and so is less destructive than the RT instability (e.g. De Young 2003). At the present time, there are no relevant astrophysical codes that are capable of handling mixing in a satisfactory manner. On the issue of magnetic stability, more sophisticated treatments using MHD have been attempted, but the main conclusions appear to be contradictory (Konz et al. 2002; Gregori et al. 1999). We are not aware of MHD codes that are sufficiently capable of answering this question at the present time.

Without excessive erudition, which is inappropriate for a conference proceeding, it is difficult to mount a solid case for why hydro processes could ultimately save the day for HVCs. But the fact of the matter is that fast-moving gas clouds do survive their passage through the Galactic halo. These may be mostly shortlived entities on the road to destruction, suggesting that there is a largely hidden plasma component that we have yet to fully comprehend. This will require more extensive observations at difficult parts of the observational parameter space, matched by hydro codes that can properly treat instabilities and mixing in a multi-phase gas.

\section{Acknowledgements}

JBH is supported by a Federation Fellowship through the Australian Research Council. I thank Alice Quillen and Ralph Sutherland for their role in the work presented here. I acknowledge 
helpful discussions with Bob Benjamin, Chris Flynn, Bryan Gaensler, Greg Madsen and Mary Putman.

\section{References}

Agertz, O., et al. 2007, MNRAS, 380, 963

Bekki, K. \& Freeman, K. C. 2003, MNRAS, 346, L11

Benjamin, R. \& Danly, L. 1997, ApJ, 481, 764

Besla, G. et al. 2007, ApJ, 668, 949

Binney, J. \& Dehnen, W. 1997, MNRAS

Binney, J., Dehnen, W., \& Bertelli, G. 2000, MNRAS, 318, 658

Bland-Hawthorn et al. 1998, MNRAS, 299, 611

Bland-Hawthorn, J., \& Maloney, P. R. 1999, ApJL, 510, L33

Bland-Hawthorn, J., \& Maloney, P. R. 2002, Extragalactic Gas at Low Redshift, 254, 267

Bland-Hawthorn, J., Sutherland, R., Agertz, O., \& Moore, B. 2007, ApJ, 670, L109

Bregman, J. N. 2007, ARA\&A, 45, 221

Brüns, C., et al. 2005, AAp, 432, 45

Canto, J. \& Raga, A. C. 1991, ApJ, 372, 646

Chandrasekhar, S. 1961, Hydrodynamic and Hydromagnetic Stability, Clarendon, Oxford

Chevalier, R. A. \& Raymond, J. C. 1978, ApJL, 225, L27

Connors, T. W., Kawata, D., \& Gibson, B. K. 2006, MNRAS, 371, 108

De Young, D. S. 2003, MNRAS, 343, 719

Ferrara, A. \& Field, G. B. 1994, ApJ, 423, 665

Flynn, C. et al. 2006, MNRAS, 372, 1149

Gaensler, B. et al. 2008, PASA, submitted

Gibson, B. K. et al. 2000, AJ, 120, 1830

Gregori, G. et al. 1999, ApJ, 527, L113

Heng, K. \& McCray, R. 2007, ApJ, 654, 923

Hibbard, J. E. \& van Gorkom, J. H. 1996, AJ, 111, 655

Hill, A. et al. 2008, ApJ, 686, 363

Jones, T. W. \& De Young, D. 2005, ApJ, 624, 586

Kahn, F. D. 1980, AAp, 83, 303

Kaiser, C. R., Pavlovski, G., Pope, E. D. C., \& Fangohr, H. 2005, MNRAS, 359, 493

Kalberla, P. \& Dedes, L. 2008, A\& A, in press (0804.4831)

Kallivayalil, N., van der Marel, R. P. \& Alcock, C., 2006, ApJ, 652, 1213

Keres, D., Katz, N., Weinberg, D. H. \& Dave, R. 2005, MNRAS, 363, 2

Klein, R. I., McKee, C. F., \& Colella, P. 1994, ApJ, 420, 213

Konz, C., Brüns, C., \& Birk, G. T. 2002, A\&A, 391, 713

Larson, R. B. 1969, MNRAS, 145, 405

Lehner, N. \& Howk, C. 2007, MNRAS, 377, 687

Liska, R. \& Wendroff, B. 1999, International Journal for Numerical Methods in Fluids, 30, 461

Lockman, F. J., Benjamin, R. A., Heroux, A. J., \& Langston, G. I. 2008, ApJ, 679, L21

Madsen, G. J., Haffner, L. M., \& Reynolds, R. J. 2002, ASPC, 276, 96

Maloney, P. R. \& Bland-Hawthorn, J. 1999, ApJL, 522, L81

Maloney, P. 1993, ApJ, 414, 41

Mastropietro, C., Moore, B., Mayer, L., Wadsley, J., \& Stadel, J. 2005, MNRAS, 363, 509

McKee, C. F. \& Cowie, L. L. 1977, ApJ, 215, 213

Miyamoto, M. \& Nagai, R. 1975, PASJ, 27, 533

Moore, B. \& Davis, M. 1994, ApJ, 270, 209

Murray, S. D., White, S. D. M., Blondin, J. M., \& Lin, D. N. C. 1993, ApJ, 407, 588

Murray, S. D. \& Lin, D. C. 2004, ApJ, 615, 586

Nicastro, F., Mathur, S., \& Elvis, M. 2008, Science, 319, 55

Oort, J. 1966, Bull. Astron. Inst. Neth., 18, 421

Pavlovski, G., Kaiser, C., Pope, E. C. D., \& Fangohr, H. 2008, MNRAS, 384, 1377 
Peek, J. E. G., Putman, M. E., \& Sommer-Larsen, J. 2008, ApJ, 674, 227

Piatek, S., Pryor, C., \& Olszewski, E. W. 2008, AJ, 135, 1024

Putman, M. E. et al. 2003, ApJ, 597, 948

Quilis, V. \& Moore, B. 2001, ApJ, 555, L95

Rasmussen, A., Kahn, S., \& Paerels, F. 2003, ASSL, 281, 109

Rosen, A. \& Smith, M. D. 2004, MNRAS, 347, 1097

Ruszkowski, M., Enslin, T. A., Bruggen, M., Heinz, S., \& Pfrommer, C. 2007, MNRAS, 378, 662

Savage, B. D. et al. 2003, ApJS, 146, 125

Sembach, K. R., Howk, J. C., Savage, B. D., Shull, J. M., \& Oegerle, W. R. 2001, ApJ, 561, 573

Sembach, K. R., et al. 2003, ApJS, 146, 165

Sembach, K. R. et al. 2004, ApJS, 150, 387

Slavin, J. D., Shull, J. M., \& Begelman, M. C. 1993, ApJ, 407, 83

Smith, M. et al. 2007, MNRAS, 379, 755

Sternberg, A., McKee, C. F., \& Wolfire, M. 2002, ApJS, 143, 419

Sternberg, A. \& Soker, N., 2008, MNRAS, 389, L13

Sun, X. H., Reich, W., Waelkens, A., \& Enslin, T. A. 2008, A\&A, 477, 573

Sutherland, R. S., 2008, ApJ, in preparation

Tamm, A., Tempel, E., \& Tenjes, P. 2007, astro-ph/0707.4375

Teyssier, R. 2002, AAp, 385, 337

Thom, C., et al. 2008, astro-ph

Tisserand, P. et al. 2007, A\&A, 469, 387

Tripp, T. M., et al. 2003, AJ, 125, 3122

Wakker, B. P. 2001, ApJS, 136, 463

Wakker, B. P et al. 2007, ApJ, 207, 670, L113

Weiner, B.J. \& Williams, T. B. 1996, AJ, 111, 1156

Weiner, B. J., Vogel, S. N., \& Williams, T. B. 2002, Extragalactic Gas at Low Redshift, 254, 256

Westmeier, T. \& Koribalski, B. S. 2008, MNRAS, 388, L29

Wilkinson, M. I. \& Evans, N. W. 1999, MNRAS, 310, 645

Williams, J. P. \& McKee, C. F. 1997, ApJ, 476, 166

Wolfire, M. et al. 1995, ApJ, 453, 673

Wolfire, M. et al. 2003, ApJ, 587, 278 